ARTICLE

\title{
A fluorescent hormone biosensor reveals the dynamics of jasmonate signalling in plants
}

\author{
Antoine Larrieu ${ }^{1,2, \star}$, Antony Champion ${ }^{3,4, \star}$, Jonathan Legrand ${ }^{1}$, Julien Lavenus ${ }^{3}$, David Mast ${ }^{1}$, \\ Géraldine Brunoud ${ }^{1}$, Jaesung $\mathrm{Oh}^{2, \dagger}$, Soazig Guyomarc'h ${ }^{5}$, Maxime Pizot ${ }^{3}$, Edward E. Farmer ${ }^{6}$, \\ Colin Turnbull7, Teva Vernoux ${ }^{1, \star}$, Malcolm J. Bennett ${ }^{2, \star} \&$ Laurent Laplaze ${ }^{3,4, \star}$
}

Activated forms of jasmonic acid (JA) are central signals coordinating plant responses to stresses, yet tools to analyse their spatial and temporal distribution are lacking. Here we describe a JA perception biosensor termed Jas9-VENUS that allows the quantification of dynamic changes in JA distribution in response to stress with high spatiotemporal sensitivity. We show that Jas9-VENUS abundance is dependent on bioactive JA isoforms, the COI1 co-receptor, a functional Jas motif and proteasome activity. We demonstrate the utility of Jas9-VENUS to analyse responses to JA in planta at a cellular scale, both quantitatively and dynamically. This included using Jas9-VENUS to determine the cotyledon-to-root JA signal velocities on wounding, revealing two distinct phases of JA activity in the root. Our results demonstrate the value of developing quantitative sensors such as Jas9-VENUS to provide high-resolution spatiotemporal data about hormone distribution in response to plant abiotic and biotic stresses.

\footnotetext{
${ }^{1}$ Laboratoire de Reproduction et Développement des Plantes, CNRS, INRA, ENS Lyon, UCBL, Université de Lyon, 69364 Lyon, France. ${ }^{2}$ Centre for Plant Integrative Biology, University of Nottingham, Nottingham LE12 5RD, UK. ${ }^{3}$ Institut de Recherche pour le Développement, Unité Mixte de Recherche Diversité Adaptation et Développement des plantes, 911 Avenue Agropolis, 34394 Montpellier, France. ${ }^{4}$ Laboratoire mixte international Adaptation des Plantes et microorganismes associés aux Stress Environnementaux, CP 18534 Dakar, Senegal. ${ }^{5}$ Université Montpellier 2, Unité Mixte de Recherche Diversité Adaptation et Développement des plantes, 34394 Montpellier, France. ${ }^{6}$ Department of Plant Molecular Biology, Université de Lausanne, 1015 Lausanne, Switzerland. ${ }^{7}$ Department of Life Sciences, Imperial College London, London SW7 2AZ, UK. * These authors contributed equally to this work. $\dagger$ Present address: Plasma Technology Research Center, National Fusion Research Institute, Gunsan-City, Republic of Korea. Correspondence and requests for materials should be addressed to L.L. (email: laurent.laplaze@ird.fr).
} 
$\mathrm{P}$ lants are subject to a variety of biotic and abiotic stresses such as the presence of pathogens, insect or mechanical injury, and several environmental stresses. These stresses lead to changes in hormone levels and gene expression that occur within minutes and activate local and systemic responses. Many of these adaptive responses are regulated by the plant hormone jasmonic acid (JA) and its derivatives, collectively referred to as jasmonates ${ }^{1}$. However, due to the current lack of tools to visualize the sites of JA perception in planta, there are major gaps in our understanding of how JA exerts its effects locally or systemically. JA signalling triggers large-scale changes in gene expression $^{2}$. In cells with a low JA content, transcription factors that regulate JA-responsive genes are repressed by members of the Jasmonate-ZIM-Domain (JAZ) protein family. The bioactive form of JA conjugated to isoleucine, termed JA-Ile, promotes the binding of JAZ repressors to the F-box protein CORONATINE INSENSITIVE1 (COI1). This F-box protein is part of the $\mathrm{SCF}^{\mathrm{COIl}} \mathrm{E}_{3}$ ubiquitin ligase complex that promotes JAZ degradation via the ubiquitin/26S proteasome pathway. Therefore, in the presence of JA-Ile, JAZ proteins are degraded and transcription factors are relieved from repression ${ }^{3-5}$. A specific Jas motif in JAZ proteins is responsible for their interaction with COI1 and their JA-dependent degradation ${ }^{6,7}$.

Biosensors are tools that transform a recognition event such as the perception of a small molecule by a receptor into a signal that can be easily detected and quantified ${ }^{8,9}$. In theory, a biosensor should (1) respond specifically to its target, (2) demonstrate a quantitative response over a physiologically relevant concentration range, (3) provide a dynamic and spatially resolved response in vivo and (4) have no impact on the endogenous signalling system it is reporting ${ }^{8,9}$. The recent development of biosensors for phytohormones such as auxin or abscisic acid (ABA) has led to significant progress in our understanding of the distribution of these molecules and their signalling mechanisms $s^{10-12}$. Here we describe a novel JA perception biosensor termed Jas9-Venus and demonstrate its utility to quantitatively and dynamically analyse JA response and distribution in planta at the cellular scale.

\section{Results}

Jas9-VENUS is a biosensor for perception of bioactive JA. Previous studies have shown that JAZ proteins such as JAI3 and JAZ1 are degraded by the proteasome pathway in response to JA, and that this degradation is dependent on a specific Jas motif in the protein $^{3,4}$. This provided a potential mechanism to develop biosensors to follow JA perception in vivo. To test this hypothesis, we generated translational fusions between the putative Jas motif of AtJAZ1 and AtJAZ9 proteins, including key amino acid residues on either side of the Jas motif ${ }^{6}$, the fast maturing VENUS variant of the yellow fluorescent protein ${ }^{13,14}$ and a N7 nuclear localization signal ${ }^{15}$ (Fig. 1a). The corresponding constructs were expressed under the control of the constitutive CaMV $35 \mathrm{~S}$ promoter and transformed into Arabidopsis thaliana plants expressing a nuclear Histone $\mathrm{H} 2 \mathrm{~B}$ marker fused to the red fluorescent protein (H2B-RFP), under the regulation of the same promoter, for ratiometric measurements ${ }^{16}$ (Supplementary Fig. 1a-c). This approach limits biases in fluorescence levels that would be due to altered regulation of the CaMV $35 \mathrm{~S}$ promoter. Robust fluorescence was only observed for Jas ${ }^{\text {JAZ9 }}$ VENUS lines, suggesting that Jas ${ }^{\mathrm{JAZ} 1}$-VENUS turnover is faster than its maturation time. This is consistent with the fast turnover observed for a JAZ1-GUS fusion ${ }^{4}$. We therefore focused our studies on Jas ${ }^{\text {JAZ9 }}$-VENUS, henceforth called Jas9-VENUS.

Next, we probed the quantitative relationship between Jas9-VENUS and JAs. We analysed changes in fluorescence in transgenic seedlings following JA treatment, using confocal microscopy. The bacterial toxin coronatine is structurally similar to JA-Ile and is a potent agonist of the JA receptor ${ }^{7}$. We observed that low levels of coronatine led to rapid degradation of Jas9VENUS in plant roots (Fig. 1b,d, Supplementary Fig. 1d and Supplementary Movie 1). Time-course imaging of roots revealed that the Jas9-VENUS signal started to decrease within minutes after coronatine treatments. Western blot analyses confirmed that the decrease in fluorescence was due to Jas9-VENUS degradation (Fig. 1c). Fluorescence quantification indicated that Jas9-VENUS degradation was dose dependent (Fig. 1d) and specifically induced by bioactive JAs (Fig. 1e) but not, or very delayed and only to a limited degree, by other hormones such as auxin, ABA, ethylene (ACC (1-aminocyclopropanecarboxylic acid), ethylene precursor) or salicylic acid (SA) (Fig. 1f). The limited and delayed degradation of Jas9-VENUS observed on treatment with other hormones is likely to result from cross-talk between these signals and their transduction pathways with JA signalling ${ }^{17}$. The JA-Ile precursors, JA and 12-oxo-phytodienoic acid (OPDA) triggered a very rapid response, indicating that these molecules quickly enter the cell and get metabolized to generate JA-Ile (Fig. 1e). Moreover, degradation of Jas9-VENUS was abolished in the coil-1 mutant background ${ }^{18}$ and strongly reduced when plants were treated with the proteasome inhibitor MG132 (Fig. 1g). A stabilized Jas9-VENUS version was created by site-directed mutagenesis on the critical amino acids $\mathrm{R}$ and $\mathrm{K}$ at position 223 and 224, respectively, of the Jas motif being substituted with alanine residues. These mutations have been demonstrated to prevent binding to COI1, and therefore JAZ proteins degradation in response to JA treatments ${ }^{19}$. This mutated version of the sensor, termed mJas9-VENUS, was not degraded in response to coronatine treatment (Fig. 1g). Altogether, these data indicate that Jas9-VENUS is a quantitative and specific sensor of jasmonate perception in planta.

Next, we compared the growth response of Jas9-VENUS and control ( Pro $\left._{35 \mathrm{~S}}: \mathrm{H} 2 \mathrm{~B}-\mathrm{RFP}\right)$ plants to coronatine. Dose-response curves revealed that Jas9-VENUS roots exhibit the same profile of root growth inhibition by coronatine as control plants (Supplementary Fig. 2a). Similarly, the induction of JA-responsive genes in response to wounding was maintained in Jas9-VENUS plants (Supplementary Fig. 2b). Hence, our data indicate that the Jas9-VENUS sensor has no detectable effect on either physiological or molecular responses to JA.

Use of Jas9-VENUS to map local changes in JA distribution. JA inhibits root growth, notably through the direct repression of PLETHORA (PLT) genes by the transcription factor MYC2 (ref. 20). However, the spatial distribution of JA in the root meristem remains unclear. We used Jas9-VENUS and H2B-RFP fluorescence to generate a map of JA distribution in the root apical meristem (Fig. 2a, Supplementary Fig. 3 and Supplementary Data 1). As Jas9-VENUS degradation is COI1 dependent, we first analysed the expression of COI1 in the root using a translational Pro COI1 $_{\text {:COI1-VENUS fusion. The }}$ construct revealed a homogenous distribution of the receptor in the majority of root cell types (Supplementary Fig. 4 and Supplementary Data 1). Slightly higher expression was detected in elongating epidermal cells and these cells might therefore be more sensitive to JA. However, the spatial pattern of Jas9-VENUS fluorescence that we observed in the root apical meristem should not be influenced by differences in JA perception, except in the mature epidermis, where potential overestimation of JA levels occurs due to high expression of COI1. Our imaging studies revealed that basal JA levels are higher in the epidermal, ground tissue, pericycle and vascular initials, and in their daughter cells in 
a

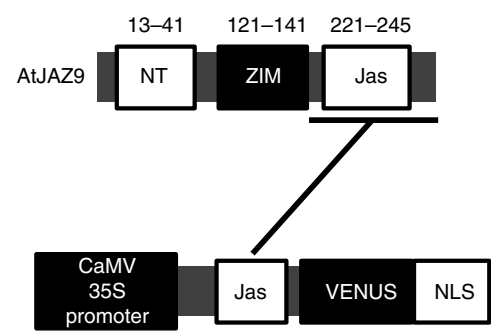

Jas9 PQARKASLARFLEKRKERLMSAMPY mJas 9 PQAAAASLARFLEKRKERLMSAMPY

d

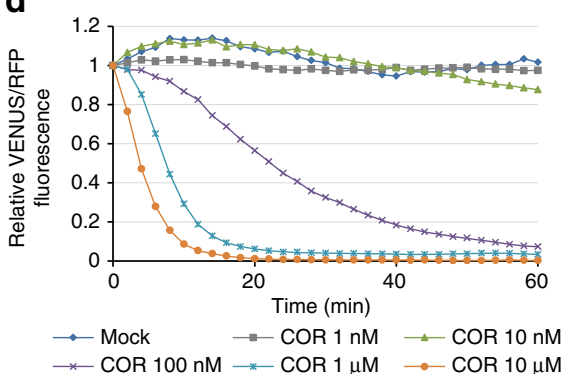

f

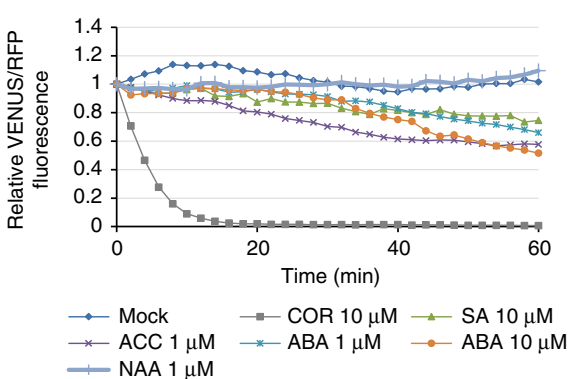

b

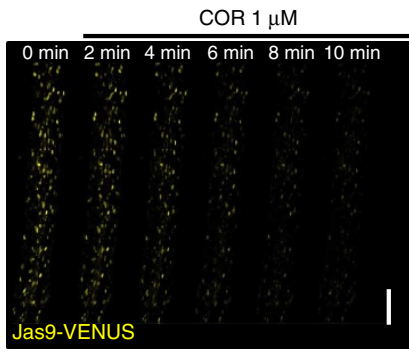

C

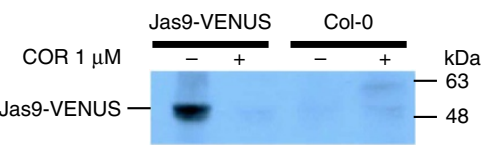

e

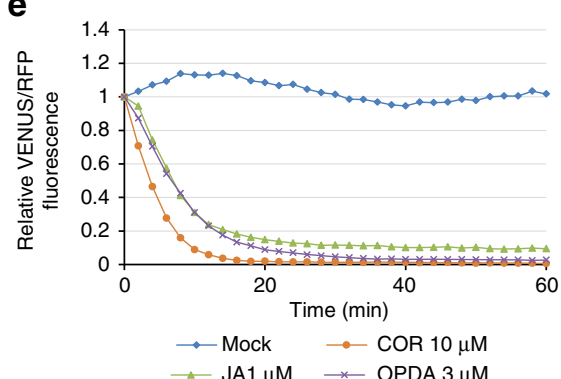

g

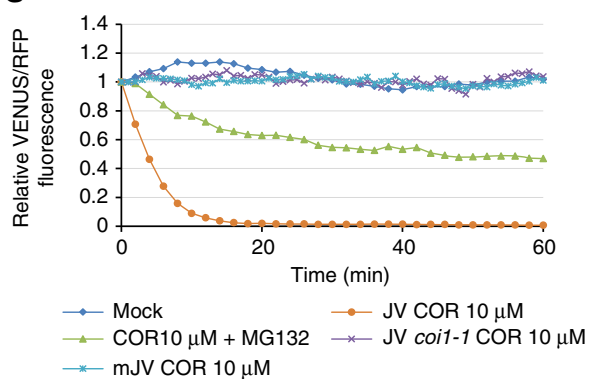

Figure 1 | Jas9-VENUS is a biosensor for bioactive JA perception. (a) Schematic representation of the AtJAZ9 gene, the Jas9-VENUS and mJas9-VENUS constructs, as well as the protein sequences of their respective Jas motif (Jas, Jas motif; NLS, nuclear localization signal; NT, amino terminus; ZIM, ZIM motif). Numbers above the schematic represent amino acid position. (b) Time-course confocal laser scanning micrographs of Jas9-VENUS at the indicated time points after treatments with $1 \mu \mathrm{M}$ coronatine (COR; scale bar, $100 \mu \mathrm{m}$ ). (c) Western blot analysis of total protein extracts of Jas9-VENUS and Col-0 seedlings treated for $30 \mathrm{~min}$ with or without $1 \mu \mathrm{M}$ COR and probed with an anti-GFP antibody (uncropped western blotting is shown in Supplementary Fig. 1d). (d) Time-course quantification of Jas9-VENUS fluorescence, normalized to H2B-RFP fluorescence, in response to various concentrations of coronatine $(n=3)$. (e) Time-course quantification of Jas9-VENUS fluorescence, normalized to H2B-RFP fluorescence, in response to JA-Ile precursors JA and OPDA $(n=2)$. (f) Time-course quantification of Jas9-VENUS fluorescence, normalized to H2B-RFP fluorescence, in response to auxin ( $\alpha$-naphthalene acetic acid (NAA)), ABA, ACC (ethylene precursor) or SA $(n=2)$. (g) Time-course quantification of Jas9-VENUS fluorescence, normalized to H2B-RFP fluorescence, in response to treatments with COR in a WT or a coil-1 mutant, in response to COR and MG132 (proteasome inhibitor) treatments, and of mJas9-VENUS fluorescence, normalized to H2B-RFP fluorescence, in response to COR $(n=2)$. All the data shown derive from a representative experiment and the number of replications of each experiment is indicated. JV, Jas9-VENUS; mJV, mJas9-VENUS.

the division zone than in other parts of the root (Fig. 2a and Supplementary Fig. 3). Interestingly, a root JA gradient was revealed with highest concentrations close to the tip, which decreased as cells move away from the stem cell niche. Low JA accumulation occurred in the quiescent centre. Considering that jasmonate is a stress-related hormone present at low concentrations in unstimulated tissues ${ }^{21}$, this suggests a role for JA in the functioning of the root apical meristem. No JA was found to accumulate in the root cap, except in last two cells of the lateral root cap. Hence, Jas9-VENUS together with the COI1 translational reporter can be used to map the distribution of JA in a given cell, tissue or organ.

Mechanical stress is also known to trigger JA production in plants $^{21}$. Therefore, we investigated the dynamics of local response of the Jas9-VENUS sensor to mechanical stress by applying a mechanical pressure to roots using an agar block. The reporter was degraded in all of the cells under pressure in $<20 \mathrm{~min}$, indicating that JA signalling was rapidly activated throughout the root (Fig. 2b). The degradation was again COI1 dependent as demonstrated by the lack of response in a coil-1 mutant background or in the mJas9-VENUS line (Fig. 2b). Similarly, perturbations in cell wall properties by genetic (mutations) or pharmacological (isoxaben) inhibition of cellulose biosynthesis lead to mechanical stress and JA production $^{22,23}$. Consistently, we observed a rapid degradation of the Jas9-VENUS sensor starting in the elongation zone around $110 \mathrm{~min}$ after isoxaben treatment and then moving to neighbouring regions of the root apex (Fig. $2 \mathrm{c}-\mathrm{e}$ and 
a

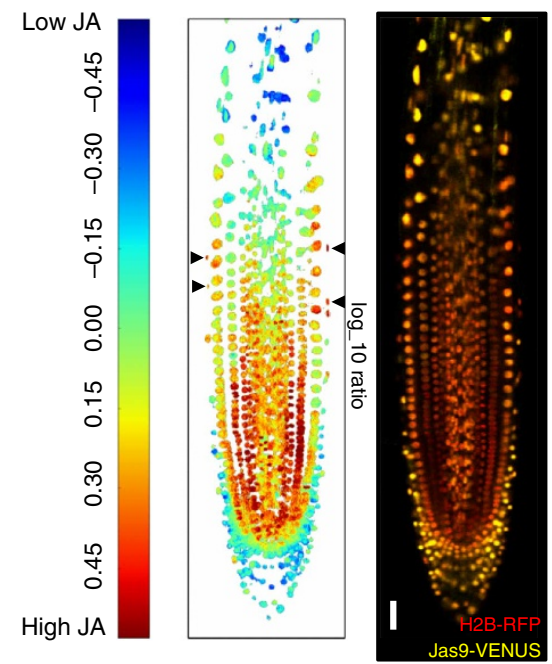

b
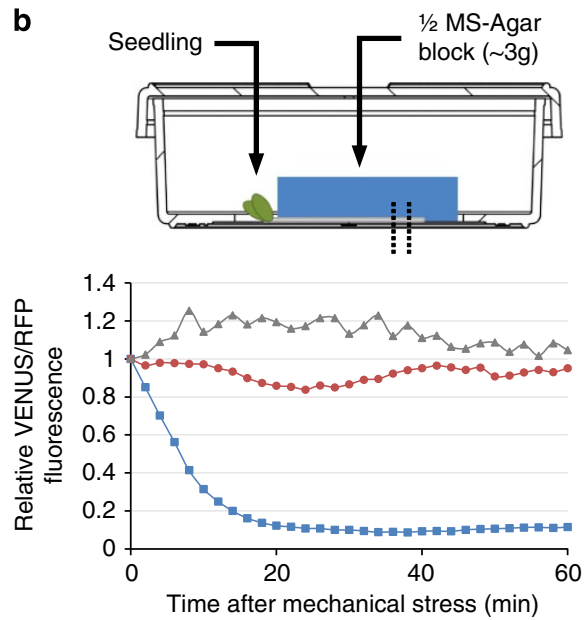

$\because \mathrm{JV} \rightarrow \mathrm{JV}$ coil $-1 \rightarrow \mathrm{mJV}$
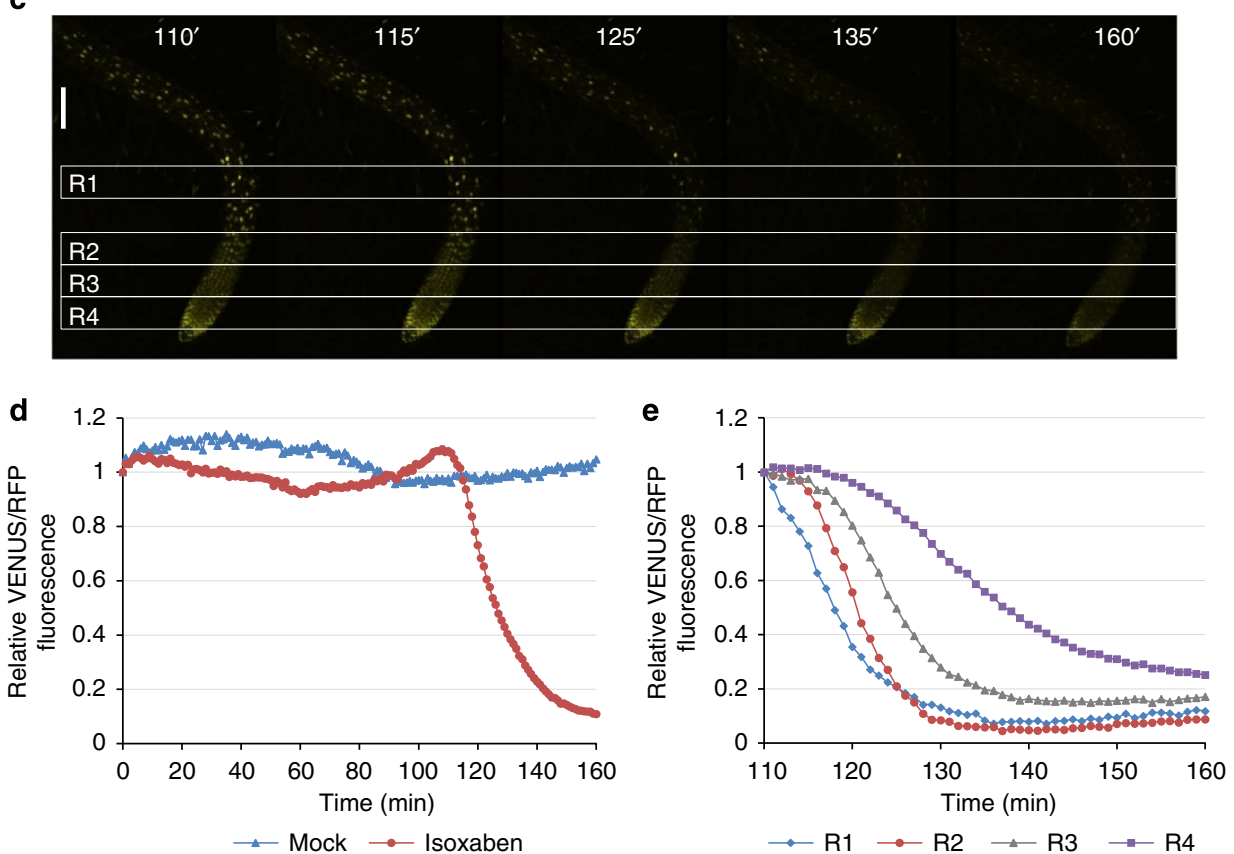

Figure 2 | Jas9-VENUS can be used to map local changes in bioactive JA. (a) Distribution map of bioactive JA in the root apical meristem based on the ratio of Jas9-VENUS fluorescence to H2B-RFP fluorescence of a representative seedling. The overlay of the Jas9-VENUS and H2B-RFP channels used for generating the root map is shown (scale, $30 \mu \mathrm{m}$ ). In total, three independent transgenic lines were analysed $(n=5)$ and representative maps are shown in Supplementary Fig. 3. The map revealed that basal JA levels are higher in the epidermal, ground tissue, pericycle and vascular initials, and in their daughter cells in the division zone than in other parts of the root. (b) Seedlings are mechanically stimulated by gently laying down a block of agar ( $\sim 3 \mathrm{~g}$ ) on the entire root. Time-course quantification of Jas9-VENUS fluorescence, normalized to H2B-RFP fluorescence, in a WT or coi1-1 mutants, and of mJas9-VENUS fluorescence, normalized to H2B-RFP fluorescence, in response to a mechanical stimulus. (c) Confocal micrographs taken at the indicated time points after treatments with $10 \mu \mathrm{M}$ isoxaben (scale bar, $100 \mu \mathrm{m}, n=2$ ). (d) Time-course quantification of Jas9-VENUS fluorescence, normalized to H2B-RFP fluorescence, over 160 min after isoxaben and mock treatments. (e) Time-course quantification of Jas9-VENUS fluorescence, normalized to H2B-RFP fluorescence, between 110 and 160 min after treatments with isoxaben for the four regions of the root indicated on the micrographs. Drawing used in $\mathbf{b}$ kindly provided by Greiner Bio-One $\mathrm{GmbH}$, Frickenhausen, Germany. All the data shown derive from a representative experiment and the number of replications of each experiment is indicated. JV, Jas9-VENUS; mJV, mJas9-VENUS.

Supplementary Movie 2). Hence, the Jas9-VENUS sensor reveals JA-Ile perception at a cellular resolution in plant roots in response to local stresses and allows the spread of the JA signal to be monitored at the tissue level.

Use of Jas9-VENUS to study long-distance JA signalling. The mechanisms by which plants perceive and respond to tissue injury are complex. Biotic or mechanical stresses lead to systemic induction of host defence responses that protects healthy tissues from secondary attack. This JA-mediated process involves longdistance communication between tissues ${ }^{24}$ and occurs within minutes of tissue injury. To analyse long-distance JA signalling, we measured VENUS fluorescence in the root following wounding of one cotyledon. We observed a rapid decrease in Jas9-VENUS fluorescence in the root (Fig. 3a, Supplementary 
a
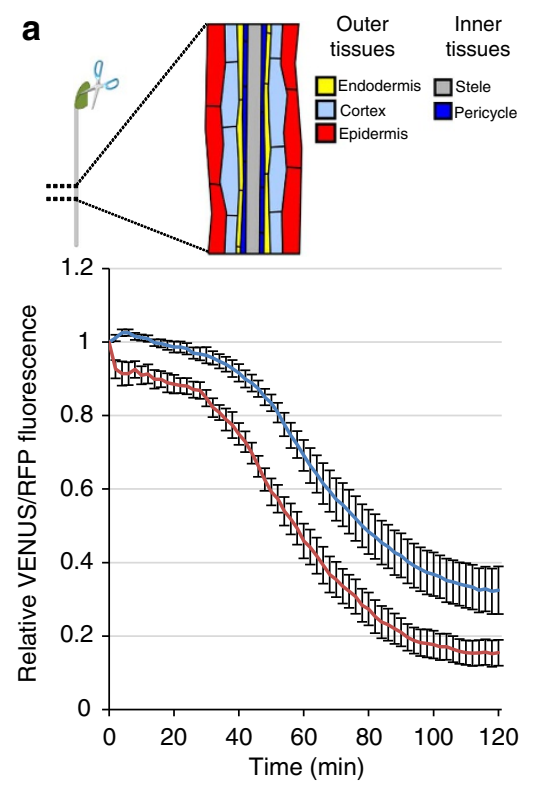

- Outer — Inner

C

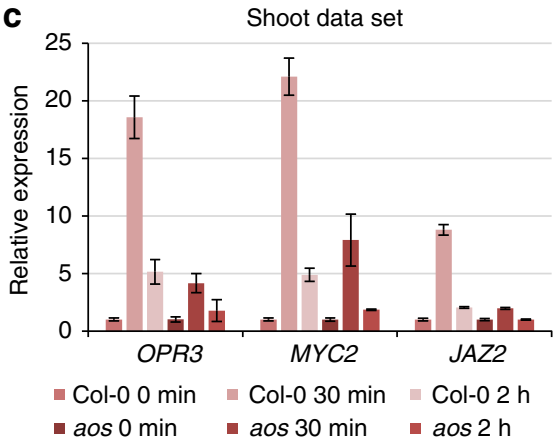

b
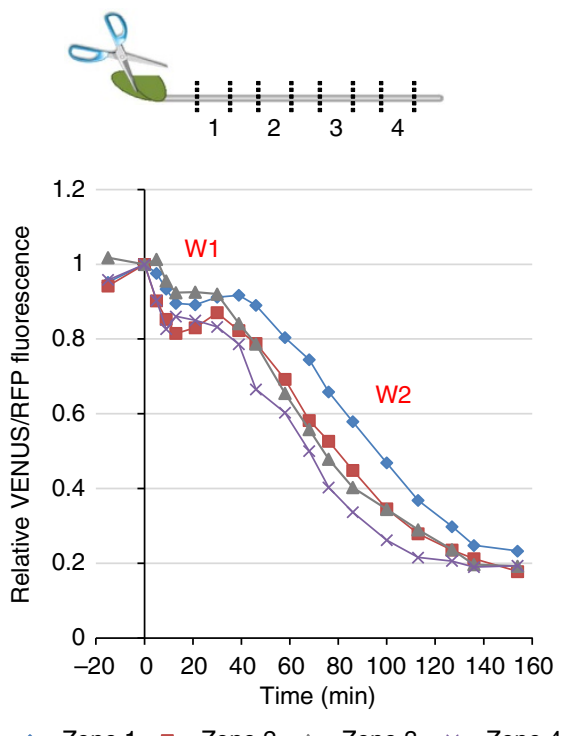

$\bullet$ Zone $1-$ Zone $2 \rightarrow$ Zone $3 \rightarrow$ Zone 4

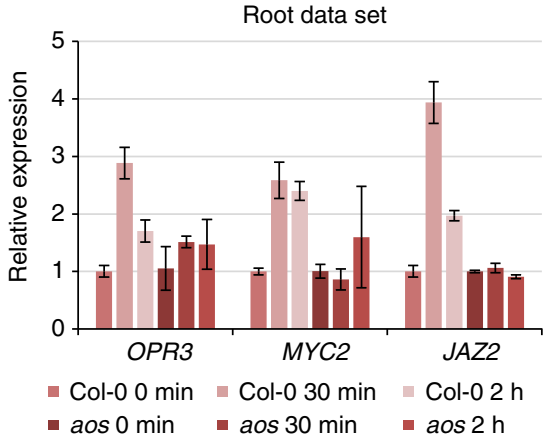

Figure 3 | Jas9-VENUS can be used to study long-distance JA signalling in planta. (a) Seedlings were wounded by cutting a cotyledon using dissection scissors. Time-course quantification of Jas9-VENUS fluorescence, normalized to H2B-RFP fluorescence, in the inner and outer tissues of the root over $120 \mathrm{~min}$ after wounding. Error bars show the s.e.m. of 13 replicates. (b) Time-course quantification of Jas9-VENUS fluorescence, normalized to H2B-RFP fluorescence, in the inner tissues at four positions along the root (indicated on the diagram) before and after wounding. (c) Shoots and roots of 1-week-old WT or aos seedlings were harvested $30 \mathrm{~min}$ and $2 \mathrm{~h}$ after wounding and the expression of wound-inducible genes were investigated using RT-qPCR. The experiment was repeated twice and the results show a representative data set. Error bars represent the s.d. of four technical replicates.

Fig. 5 and Supplementary Movie 3). In contrast to local mechanical stress, the decrease in VENUS fluorescence occurred in two distinct phases in 10 of 14 experiments (Fig. 3a and Supplementary Fig. 5). An initial phase amounting to a loss of $\sim 8 \%$ of the signal was completed $\sim 2$ min after wounding (Fig. 3a). This first phase was mainly detected in the stele, suggesting that the signal was moving in the vasculature (Fig. 3a and Supplementary Fig. 5). To determine the velocity of the signal triggered by wounding, fluorescence was measured at four different positions along the root (Fig. 3b). Interestingly, the loss of VENUS fluorescence characteristic of this first phase occurred simultaneously at the root/shoot junction (Zone 1) and in close vicinity of the root tip (Zone 4), which were $1 \mathrm{~cm}$ apart. Based on the experiment set-up, we estimate the speed of this first wave to exceed $1 \mathrm{~cm} \mathrm{~min}^{-1}$. This observation is in the same order of magnitude as measurements reported for leaf-to-leaf wound signalling ${ }^{25}$. A second phase observed in all experiments started at $\sim 30 \mathrm{~min}$ and lasted for $90 \mathrm{~min}$, leading to a further $70 \%$ decrease of the fluorescence signal. It occurred first in the stele and then in the outer tissues, and starts in the root tip region (Zone 4; Fig. 3a and Supplementary Fig. 5). Using reverse transcriptase quantitative-PCR (RT-qPCR), we confirmed that the decrease in VENUS fluorescence after wounding was not due to an altered expression of the receptor COI1 and of the sensor Jas9-VENUS, as the expression of both mRNA is stable after wounding in the shoot and in the root (Supplementary Fig. 6a,b). We also showed that the reduction in VENUS fluorescence in response to wounding required a functional Jas motif (Supplementary Fig. 7). Thus, the Jas9-VENUS reporter revealed that a two-step JA response is induced in the root in response to wounding in the aerial part of the plant and allows the estimation of the speed of the signal triggering this response.

We investigated whether the response observed with the Jas9VENUS sensor could be correlated with documented JA responses. Roots and aerial parts from Arabidopsis plants were harvested before and $30 \mathrm{~min}$ and $2 \mathrm{~h}$ after wounding a cotyledon, and the expression of genes induced by JA was measured by RTqPCR. We observed a rapid induction of JA-responsive genes in roots after wounding in accordance with the rapid response of the Jas9-VENUS reporter (Fig. 3c). This induction is dependent on JA production, as the biosynthesis mutant $\operatorname{aos}^{26}$ exhibited little or no induction of those genes (Fig. 3c). Moreover, JA was recently 
shown to stabilize the JA-responsive MYC2 transcription factor ${ }^{27}$. Accordingly, we observed that a stabilization of a MYC2-GFP fusion protein in the root $1 \mathrm{~h}$ after wounding the cotyledon (Supplementary Fig. 8). This stabilization was lost in a MYC2 $\triangle D E-G F P$ construct that was shown to be unable to respond to $\mathrm{JA}^{27}$. This is consistent with an induction of local JA production in the root corresponding to the second phase of the response detected with Jas9-VENUS. Altogether, gene expression data and MYC2 protein stabilization are consistent with the dynamics of JA signalling that we observed with the Jas9-VENUS sensor.

\section{Discussion}

Here we describe Jas9-VENUS, a new tool to monitor the perception of biologically active jasmonates in planta. We show that Jas9-VENUS is specifically and rapidly degraded in the presence of bioactive JA in a dose-dependent manner. Moreover, we demonstrate that Jas9-VENUS degradation is dependent on COI1 and the proteasome. Finally, our results indicate that the Jas9-VENUS construct does not perturb JA perception and responses. Altogether, Jas9-VENUS has all the attributes of a JA perception biosensor, that is, its fluorescence is directly correlated to the combinatorial action of the signal and the receptor machinery.

Our results suggest that Jas9 might be more stable than other Jas motifs such as Jas1 and might therefore not be as responsive for fast JA responses or suitable to calculate velocity for propagation of rapid JA responses. However, in practice, Jas9VENUS degradation was dose dependent and responded very rapidly (in a few minutes) to JA. Moreover, analyses of JAresponsive gene expression are consistent with the observed dynamics of Jas9-VENUS degradation. Jas9-VENUS thus offers a good compromise between a strong fluorescence signal, sensitivity, specificity and a quantitative and rapid response, as expected from a genetically encoded sensor ${ }^{9}$. In addition, we used of a ratiometric approach combining the Jas9-VENUS construct to a nuclear-localized 35S-H2B:RFP construct, to prevent errors due to a transcriptional effect on the $35 \mathrm{~S}$ promoter. However, we cannot rule out that the use of two different constructs containing the $35 \mathrm{~S}$ promoter could still induce biases in ratio quantification due to genomic position effects that might have an impact on the expression of the transgene or to silencing. Ideally, both Jas9Venus and $\mathrm{H} 2 \mathrm{~B}-\mathrm{RFP}$ would be expressed from the same promoter as a single protein that would be cleaved to avoid these problems ${ }^{28}$. Such a design feature would prove useful in future biosensors development.

Our studies of local and long-distance JA responses showed the value of Jas9-VENUS to image the quantitative dynamics of these responses at a high temporal and spatial resolution. Using Jas9VENUS, we were able to generate a map of JA accumulation in the root apical meristem. Exogenous JA is known to regulate root growth $^{1}$ and jasmonates could mediate root response to various biotic and abiotic environmental signals. Here we show that JA accumulates in the epidermis, ground tissues and vascular tissue from the initials to the end of the division zone in undamaged plants. This is coherent with the expression of the JA-inducible ASA1 gene in the root tip ${ }^{29}$. ASA 1 encodes an enzyme involved in auxin biosynthesis and this JA-auxin cross-talk could mediate some of the JA-regulated root responses to environmental signals. Moreover, our map revealed JA accumulation in the outer lateral root cap and in the epidermis. This is in agreement with previously reported map of JA-related gene expression in the root apical meristem ${ }^{30}$, suggesting that JA distribution might be the primary determinant of JA-induced gene expression in this tissue. Interestingly, outer lateral root cap cells were shown to enter programmed cell death ${ }^{31}$. Although the role of JA in programmed cell death has been documented in other systems ${ }^{32}$, this suggests a novel role for JA in regulating lateral root cap development. Finally, the high levels of JA detected in the ground tissue initials and daughter cells suggest an unanticipated role for JA in the activity of the root apical meristem.

We also used the Jas9-VENUS sensor to analyse long-distance signalling between the aerial parts and the root following a wound. It revealed that a two-step JA response is induced in the root in response to wounding in the aerial part of the plant. We first observed a rapid signal $\left(>1 \mathrm{~cm} \mathrm{~min}^{-1}\right)$ moving through the stele and inducing a limited degradation of Jas9-VENUS presumably through a limited JA production. This first phase was relatively weak and localized in the stele but robust enough to be detected in 10 out of 14 experiments. Moreover, the variability of response we observed could also result from the difficulties of performing wounding in a reproducible way. In leaves, this fastmoving signal is probably related to the membrane depolarization occurring in sieve elements in response to wounding and responsible for leaf-to-leaf signalling ${ }^{25,33}$. A similar mechanism may participate in leaf-to-root signalling and might induce the very rapid conversion of a local pool of inactive jasmonate precursors into active JA. This would in turn trigger the expression of JA biosynthesis genes and therefore strongly enhance JA accumulation that would contribute to the second phase of the response. This biphasic mechanism would therefore serve to amplify an initial rapid systemic signal to induce a later, stronger local response in the root. Electrical activity in the vasculature is probably not the only source of signals that lead to jasmonate production in wounded leaves ${ }^{25}$ and it is possible that other mechanisms underlie the second, stronger phase of jasmonate signalling that we observed in roots. Importantly, our results suggest that wounding not only induces systemic defence in the leaves but is also likely to modulate root development, physiology and defence responses.

Jasmonate is a fundamental regulator of plant responses to various abiotic and biotic stresses. The Jas9-VENUS sensor paves the way to a better characterization of the JA signalling pathway in planta. Our results further illustrate how fluorescent sensors can be used to perform multi-scale analyses of the distribution of small signalling molecules.

\section{Methods}

Chemicals and hormones. Coronatine, JA, SA, $\alpha$-naphthalene acetic acid, ACC (ethylene precursor), ( \pm )-ABA, MG132 and isoxaben were purchased from Sigma OPDA was purchased from Cayman Chemical (Keystone, Colorado, USA). Coronatine, SA, $\alpha$-naphthalene acetic acid, MG132 and isoxaben were dissolved in $100 \%$ dimethyl sulfoxide, ACC was dissolved in double distilled $\mathrm{H}_{2} \mathrm{O}$, ABA in $100 \%$ methanol and JA in $100 \%$ ethanol. All compounds were dissolved to a stock concentration of 50 or $100 \mathrm{mM}$. OPDA was purchased diluted in $100 \%$ ethanol (100 $\mu$ g per $100 \mu \mathrm{l} \sim 3.4 \mathrm{mM}$ ).

Arabidopsis lines. The JA biosynthetic mutant $a o s^{26}$ and the JA response mutant coil-1 (ref. 18) were obtained from the Nottingham Arabidopsis Centre. The p35S:H2B-RFP, p35S:MYC2-GFP and p35S:MYC2 $\Delta$ DE-GFP line were described previously ${ }^{16,27}$.

Seed sterilization and plant growth. Seeds were surface sterilized for $5 \mathrm{~min}$ in $10 \%$ bleach, $0.1 \%$ Triton X-100, then washed three times with sterile double distilled $\mathrm{H}_{2} \mathrm{O}$. Seeds were stratified at $4^{\circ}$ for 2 days to synchronize germination. For time-lapse imaging on the confocal microscope (treatments and wound responses), sterile seeds were sown directly onto glass-bottom petri dishes (ref 627861 from Greiner (Germany), and ref 3930-035 from Iwaki (Japan)) against a block of $1 / 2$ Murashige and Skoog medium (2.17 g MS salts per litre (Sigma)) at pH 5.7 solidified with 1\% bacto-agar (Appleton Woods; as shown in Supplementary Fig. 9) Seedlings were grown under continuous light $\left(100 \mu \mathrm{E} \mathrm{m}^{-2} \mathrm{~s}^{-1}\right)$. For all other experiments (mechanical stress, bulk up and gene expression), sterile seeds were sown onto round or square petri dishes containing MS agar media as described above. 
Jas9-VENUS constructs. The Jas motif from AtJAZ9 was amplified using primer JAZ9-F2 and JAZ9-R1 by PCR using complementary DNA produced from 2-week-old Arabidopsis plants treated with $50 \mu \mathrm{M}$ JA during $1 \mathrm{~h}$. The mutated version of the Jas9-VENUS sensor was generated by PCR using primers JAZ9-F3m and JAZ9-R3m. PCR products were cloned into pENTR-D TOPO (Invitrogen). The Jas motif sequence or the mutated version were then fused in-frame to VENUS-N7 under the control of the cauliflower mosaic virus $35 \mathrm{~S}$ promoter in the pH7m34GW binary plasmid ${ }^{34}$, using the Multisite Gateway three-fragment vector construction kit (Invitrogen). Plasmid constructs were confirmed by sequencing. The vectors were transformed into Agrobacterium tumefaciens strain GV3101 and into Arabidopsis p35S-H2B:RFP (Col-0 ecotype) using the floral dip transformation protocol. Lines with single insertions were selected based on the segregation of antibiotic resistance and fluorescence. Line 6 was used in most experiments unless specifically stated.

COI1 constructs. COI1 genomic sequence and its promoter were amplified by PCR using COI1-Fw and COI1-Rev-Full primers. The PCR product was purified and cloned into the entry clone pENTR-D-TOPO using TOPO cloning (Invitrogen), according to the manufacturer's instructions. The fast-maturing yellow fluorescent protein VENUS was added immediately before the STOP codon using multi-site gateway cloning into the destination vector $\mathrm{pK} 7 \mathrm{~m} 34 \mathrm{GW}$, to create the

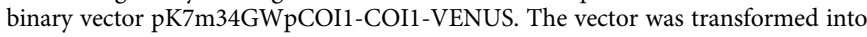
Agrobacterium strain GV3101 and into Arabidopsis Col-0 ecotype using the floral dip transformation protocol $^{35}$

Arabidopsis protein extraction. Extraction of soluble proteins was performed on 1-week-old seedlings grown on $1 / 2 \mathrm{MS}+$ agar plates. Seedlings were treated continuously with liquid $1 / 2 \mathrm{MS}$ medium for 30 min with $1 \mu \mathrm{M}$ coronatine or with dimethyl sulfoxide (Mock). Seedlings were harvested, frozen in liquid nitrogen and then ground using a Qiagen Tissue Lyser II $(30 \mathrm{~Hz}$ during $30 \mathrm{~s})$. The powder was resuspended in SDG buffer $(0.0625 \mathrm{M}$ Tris- $\mathrm{HCl} \mathrm{pH} 6.8,2.5 \% \mathrm{SDS}, 2 \%$ dithiothreitol $(0.13 \mathrm{M}), 20 \%$ glycerol) and subjected to three cycles of freezing/thawing in liquid nitrogen. Proteins were boiled for $5 \mathrm{~min}$ at $95^{\circ} \mathrm{C}$, followed by two successive rounds of centrifugation $\left(20 \mathrm{~min}, 18,000 \mathrm{~g}\right.$ at $4^{\circ} \mathrm{C}$ then $5 \mathrm{~min}, 18,000 \mathrm{~g}$ at $4^{\circ} \mathrm{C}$ ) to remove cell debris.

Western blotting. The samples were boiled for $5 \mathrm{~min}$ at $95^{\circ} \mathrm{C}$ before being loaded onto a gel (10 $\mu \mathrm{l}$ per lane). SDS-PAGE was performed on $10 \%$ polyacrylamide gel. After transfer onto a nitrocellulose membrane, protein amounts in each lane were checked using Ponceau staining. Immunoblotting analysis was realized using a primary rabbit polyclonal anti-GFP antibody (ab290, Abcam, diluted 1:5,000) and a secondary anti-rabbit Ig-HRP (NA934-1ML, Amersham, diluted 1:10,000). Proteins were visualized using the enhanced chemiluminescence kit (Amersham).

Confocal microscopy. Jas9-VENUS seedlings were imaged on an inverted Leica SP5 confocal laser scanning microscope (Leica Microsystems, Germany) or an inverted Nikon Eclipse Ti-U confocal microscope (Nikon, Japan). Scanner and detectors settings used for one experiment were optimized to avoid saturation and to maximize resolution and kept unchanged throughout the experiment. VENUS was excited using the $514 \mathrm{~nm}$ line (Leica SP5) or the $488 \mathrm{~nm}$ line (Nikon Eclipse) of an argon laser. VENUS fluorescence was collected from 520 to $560 \mathrm{~nm}$ (using the AOBS of the SP5) or using a 515/30 detector (Nikon Eclipse). RFP was excited using a 561-nm laser diode (SP5) or the 543-nm line of a Green He/Ne laser (Nikon Eclipse). RFP fluorescence was collected from 590 to $680 \mathrm{~nm}$ (using the AOBS of the SP5) or using a 605/75 detector (Nikon Eclipse).

Images were taken with a fixed delay of 2 min over a minimum time of $1 \mathrm{~h}$ using the $\times 10$ objective, to maximize the number of nuclei being observed. Initially, seedlings were imaged following the protocol described ${ }^{16}$. Briefly, seedlings were grown onto $1 / 2 \mathrm{MS}$-agar round or square petri dishes and gently transferred onto glass-bottom petri dishes. A small block of $1 \frac{1}{2}$ MS agar was slowly dropped onto the seedling and fluorescence was followed over time just above the elongation zone of the root. Initial experiments showed that such treatment induced the degradation of the Jas9-VENUS reporter. Subsequently, seeds were directly germinated onto the glass-bottom petri dishes (as shown on Supplementary Fig. 9) and roots that grew in between the agar block and the glass bottom were imaged. In such configuration, a stable VENUS fluorescence could be followed over time in the absence of treatments, although the signal fluctuated over time $( \pm 20 \%)$. Treatments were provided by adding liquid $1 / 2$ MS solution ( + indicated chemicals) at the same temperature next to the seedling that was going to be imaged. Mock treatments resulted in no major changes in fluorescence (comparable to no addition of liquid medium).

Other markers (pCOI1:COI1-VENUS, p35S:MYC2-GFP and p35S:MYC2 $\triangle D E-$ GFP) and images for the JA and COI1 root maps were imaged on an inverted Zeiss 710 confocal laser scanning microscope. Scanner and detector settings used for one experiment were optimized to avoid saturation and to maximize resolution, and kept unchanged throughout the experiment. VENUS was excited using the 514-nm line of an argon laser and collected from 520 to $560 \mathrm{~nm}$. Green fluorescent protein (GFP) was excited using a 488-nm line of an argon laser and collected from 495 to $550 \mathrm{~nm}$.
Fluorescence quantification. Fluorescence quantification was performed as described ${ }^{36}$. Briefly, background fluorescence was removed using a threshold and only fluorescence coming from the nuclei was quantified. Plots presented show changes in raw integrated density values over time, measured using FIJI software. Ratios (raw integrated density of the VENUS channel divided by the raw integrated density of the red channel) were calculated using Microsoft Excel software.

JA and COI1 root maps. The acquired confocal images were imported for processing using Python programming language. Images with the lowest saturation possible were used to avoid computational artefacts and to preserve a good signalto-noise ratio. Background (shown in white) was removed for the JA root maps by thresholding the H2B-RFP fluorescence to an intensity above 34 and for the COI1 root map by thresholding the COI1-VENUS fluorescence to an intensity above 16 . For the JA root map, the ratiometric computation is the ratio of Jas9-VENUS/H2BRFP fluorescence. To enhance the 'high JA' and 'low JA' differences, the obtained ratiometric values were transformed into a $\log _{10}$ scale. For the COI1 root map, the COI1-VENUS fluorescence values were centred around the mean to enhance for differences in fluorescence.

Reverse transcriptase quantitative-PCR. One-week-old seedlings were wounded by cutting a cotyledon. RNA was extracted from shoots and root separately using the Spectrum Plant Total RNA kit (Sigma). Poly(dT) cDNA was prepared from $1 \mu \mathrm{g}$ of total RNA with Superscript III reverse transcriptase (Invitrogen) and analysed on a StepOnePlus apparatus (Life Technologies) with the SYBR Green PCR Master Mix (Applied Biosystem), according to the manufacturer's instructions. Targets genes were quantified with specific primer pairs designed with the Universal Probe Library Assay Design Center (Roche Applied Science). All reactions were done in quadruplicate and data were analysed with Microsoft Excel 2007 (Microsoft Corporation, Redmond, USA). Expression levels were normalized to At1G04850 (CTRL1) and calibrated to unwounded seedling using the $\Delta \Delta \mathrm{Ct}$ method.

\section{References}

1. Wasternack, C. \& Hause, B. Jasmonates: biosynthesis, perception, signal transduction and action in plant stress response, growth and development. An update to the 2007 review in Annals of Botany. Ann. Bot. 111, 1021-1058 (2013).

2. Pauwels, L., Inzé, D. \& Goossens, A. Jasmonate-inducible gene: What does it mean? Trends Plant Sci. 14, 87-91 (2009).

3. Chini, A. et al. The JAZ family of repressors is the missing link in jasmonate signalling. Nature 448, 666-671 (2007)

4. Thines, B. et al. JAZ repressor proteins are targets of the SCFCOI1 complex during jasmonate signalling. Nature 448, 661-665 (2007).

5. Pauwels, L. \& Goossens, A. The JAZ proteins: a crucial interface in the jasmonate signaling cascade. Plant Cell Online 23, 3089-3100 (2011).

6. Sheard, L. B. et al. Jasmonate perception by inositol-phosphate-potentiated COI1-JAZ co-receptor. Nature 468, 400-405 (2010).

7. Yan, J. et al. The Arabidopsis CORONATINE INSENSITIVE1 protein is a jasmonate receptor. Plant Cell Online 21, 2220-2236 (2009).

8. Wells, D. M., Laplaze, L., Bennett, M. J. \& Vernoux, T. Biosensors for phytohormone quantification: challenges, solutions, and opportunities. Trends Plant Sci. 18, 244-249 (2013).

9. Sadanandom, A. \& Napier, R. M. Biosensors in plants. Curr. Opin. Plant Biol 13, 736-743 (2010).

10. Brunoud, G. et al. A novel sensor to map auxin response and distribution at high spatio-temporal resolution. Nature 482, 103-106 (2012).

11. Jones, A. M. et al. Abscisic acid dynamics in roots detected with genetically encoded FRET sensors. eLife 3, e01741 (2014).

12. Waadt, R. et al. FRET-based reporters for the direct visualization of abscisic acid concentration changes and distribution in Arabidopsis. eLife 3, e01739 (2014).

13. Shaner, N. C., Steinbach, P. A. \& Tsien, R. Y. A guide to choosing fluorescent proteins. Nat. Methods 2, 905-909 (2005).

14. Nagai, T. et al. A variant of yellow fluorescent protein with fast and efficient maturation for cell-biological applications. Nat. Biotechnol. 20, 87-90 (2002).

15. Cutler, S. R., Ehrhardt, D. W., Griffitts, J. S. \& Somerville, C. R. Random GFP::cDNA fusions enable visualization of subcellular structures in cells of Arabidopsis at a high frequency. Proc. Natl Acad. Sci. USA 97, 3718-3723 (2000).

16. Federici, F., Dupuy, L., Laplaze, L., Heisler, M. \& Haseloff, J. Integrated genetic and computation methods for in planta cytometry. Nat. Methods 9, 483-485 (2012).

17. Song, S., Qi, T., Wasternack, C. \& Xie, D. Jasmonate signaling and crosstalk with gibberellin and ethylene. Curr. Opin. Plant Biol. 21C, 112-119 (2014).

18. Xie, D.-X., Feys, B. F., James, S., Nieto-Rostro, M. \& Turner, J. G. COI1: an Arabidopsis gene required for jasmonate-regulated defense and fertility. Science 280, 1091-1094 (1998) 
19. Melotto, M. et al. A critical role of two positively charged amino acids in the Jas motif of Arabidopsis JAZ proteins in mediating coronatine- and jasmonoyl isoleucine-dependent interactions with the COI1 F-box protein. Plant J. Cell Mol. Biol. 55, 979-988 (2008).

20. Chen, Q. et al. The basic helix-loop-helix transcription factor MYC2 directly represses PLETHORA expression during jasmonate-mediated modulation of the root stem cell niche in Arabidopsis. Plant Cell 23, 3335-3352 (2011).

21. Glauser, G. et al. Spatial and temporal dynamics of jasmonate synthesis and accumulation in Arabidopsis in response to wounding. J. Biol. Chem. 283, 16400-16407 (2008)

22. Ellis, C., Karafyllidis, I., Wasternack, C. \& Turner, J. G. The Arabidopsis mutant cev1 links cell wall signaling to jasmonate and ethylene responses. Plant Cell 14, 1557-1566 (2002).

23. Desprez, T. et al. Resistance against herbicide isoxaben and cellulose deficiency caused by distinct mutations in same cellulose synthase isoform CESA6. Plant Physiol. 128, 482-490 (2002).

24. Prime-A-Plant Group et al. Priming: getting ready for battle. Mol. Plant Microbe Interact. 19, 1062-1071 (2006).

25. Mousavi, S. A. R., Chauvin, A., Pascaud, F., Kellenberger, S. \& Farmer, E. E. GLUTAMATE RECEPTOR-LIKE genes mediate leaf-to-leaf wound signalling. Nature 500, 422-426 (2013).

26. Park, J.-H. et al. A knock-out mutation in allene oxide synthase results in male sterility and defective wound signal transduction in Arabidopsis due to a block in jasmonic acid biosynthesis. Plant J. Cell Mol. Biol. 31, 1-12 (2002).

27. Zhai, Q. et al. Phosphorylation-coupled proteolysis of the transcription factor MYC2 is important for jasmonate-signaled plant immunity. PLoS Genet. 9, e1003422 (2013)

28. Wend, S. et al. A quantitative ratiometric sensor for time-resolved analysis of auxin dynamics. Sci. Rep. 3, 2052 (2013).

29. Sun, J. et al. Arabidopsis ASA1 is important for jasmonate-mediated regulation of auxin biosynthesis and transport during lateral root formation. Plant Cell 21, 1495-1511 (2009)

30. Birnbaum, K. et al. A gene expression map of the Arabidopsis root. Science 302, 1956-1960 (2003).

31. Fendrych, M. et al. Programmed cell death controlled by ANAC033/ SOMBRERO determines root cap organ size in Arabidopsis. Curr. Biol. CB 24, 931-940 (2014).

32. Reinbothe, C., Springer, A., Samol, I. \& Reinbothe, S. Plant oxylipins: role of jasmonic acid during programmed cell death, defence and leaf senescence. FEBS J. 276, 4666-4681 (2009).

33. Salvador-Recatalà, V., Tjallingii, W. F. \& Farmer, E. E. Real-time, in vivo intracellular recordings of caterpillar-induced depolarization waves in sieve elements using aphid electrodes. New Phytol. 203, 674-684 (2014).

34. Karimi, M., Bleys, A., Vanderhaeghen, R. \& Hilson, P. Building blocks for plant gene assembly. Plant Physiol. 145, 1183-1191 (2007).
35. Clough, S. J. \& Bent, A. F. Floral dip: a simplified method for Agrobacteriummediated transformation of Arabidopsis thaliana. Plant J. Cell Mol. Biol. 16, 735-743 (1998).

36. Larrieu, A. P., French, A. P., Pridmore, T. P., Bennett, M. J. \& Wells, D. M Time-profiling fluorescent reporters in the Arabidopsis root. Methods Mol. Biol. 1056, 11-17 (2014).

\section{Acknowledgements}

We thank Professor C. Li (Chinese Academy of Science) for kindly providing the 35S:MYC2-GFP-12 seeds and Dr Tara Holman for the CTRL1 primer sequence. We acknowledge the support of the Région Languedoc-Roussillon ('Chercheur d'Avenir' grant to S.G. and L.L.), a Pludisciplinary Research Grant from the Université Montpellier 2 (S.G. and L.L.), the Agropolis Fondation 'Rhizopolis' Federative Grant (J.L., S.G. and L.L.) and Agence National de la Recherche grant ANR-12-BSV6-0005 grant (A.L. and

T.V.). A.L. and M.J.B. acknowledge the support of the Biotechnology and Biological Sciences Research Council and Engineering and Physical Sciences Research Council funding to the Centre for Plant Integrative Biology. We also acknowledge funding in the form of a Biotechnology and Biological Sciences Research Council Professorial Research Fellowship (to M.J.B.) as well as funding in the form of a Biotechnology and Biological Sciences Research Council grant (A.L. and M.J.B.).

\section{Author contributions}

A.L., A.C., E.E.F., C.T., T.V., M.J.B. and L.L. designed the experiments. A.L., A.C., J.L., J.L., D.M., G.B., J.O., S.G. and M.P. performed the experiments. A.L., A.C., T.V., M.J.B., and L.L. wrote the manuscript. all the authors read and edited the final manuscript. T.V., M.J.B. and L.L. supervised the project.

\section{Additional information}

Supplementary Information accompanies this paper at http://www.nature.com/ naturecommunications

Competing financial interests: The authors declare no competing financial interests.

Reprints and permission information is available online at http://npg.nature.com/ reprintsandpermissions/

How to cite this article: Larrieu, A. et al. A fluorescent hormone biosensor reveals the dynamics of jasmonate signalling in plants. Nat. Commun. 6:6043 doi: $10.1038 /$ ncomms7043 (2015).

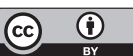

This work is licensed under a Creative Commons Attribution 4.0 International License. The images or other third party material in this article are included in the article's Creative Commons license, unless indicated otherwise in the credit line; if the material is not included under the Creative Commons license, users will need to obtain permission from the license holder to reproduce the material. To view a copy of this license, visit http://creativecommons.org/licenses/by/4.0/ 


\title{
Corrigendum: A fluorescent hormone biosensor reveals the dynamics of jasmonate signalling in plants
}

\author{
Antoine Larrieu, Antony Champion, Jonathan Legrand, Julien Lavenus, David Mast, Géraldine Brunoud, \\ Jaesung Oh, Soazig Guyomarc'h, Maxime Pizot, Edward E. Farmer, Colin Turnbull, Teva Vernoux, \\ Malcolm J. Bennett \& Laurent Laplaze
}

Nature Communications 6:6043 doi: 10.1038/ncomms7043 (2015); Published 16 Jan 2015; Updated 10 Feb 2016

It has come to our attention that the control version of the Jas9-VENUS reporter construct reported in this Article, mJas9-VENUS, which consists of a mutated version of Jas9 fused to VENUS, does not contain a nuclear localization signal as described in Fig. 1a. The conclusion that degradation of the Jas9-VENUS reporter is dependent on jasmonate signalling in the nucleus remains unaffected by this error, as the reporter construct itself contains a nuclear localization signal, and the mJas9-VENUS control construct also localizes to the nucleus, even in the absence of this sequence (Fig. 4). As a result we do not foresee any practical implications for the use of either the Jas9-VENUS sensor construct or the mJas9-VENUS control.

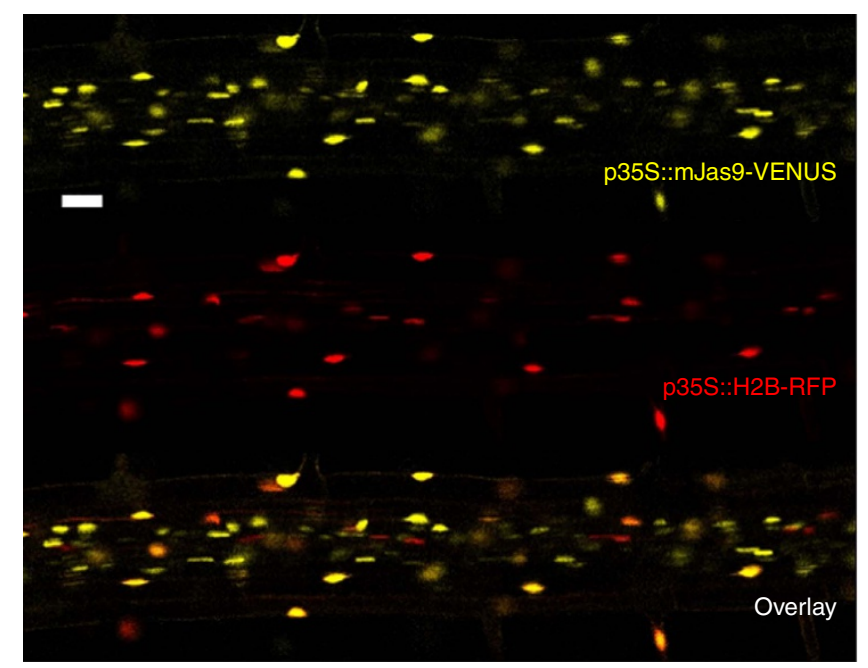

Figure 4 | mJas9-VENUS localizes to the nucleus in the mature part of the root. Images show the mature root of a 5-day-old Arabidopsis seedling expressing the nuclear marker H2B-RFP and the mJas9-VENUS control reporter construct. Scale bar, $20 \mu \mathrm{m}$.

(i) This work is licensed under a Creative Commons Attribution 4.0 International License. The images or other third party material in this article are included in the article's Creative Commons license, unless indicated otherwise in the credit line; if the material is not included under the Creative Commons license, users will need to obtain permission from the license holder to reproduce the material. To view a copy of this license, visit http://creativecommons.org/licenses/by/4.0/ 